\section{rev Psi}

Revista de Psicología (UNLP)

https://revistas.unlp.edu.ar/revpsi

\title{
Contribuciones desde la experiencia musical al debate sobre la naturaleza de la mente
}

\author{
Favio Shifres ${ }^{1}$ \\ Correspondencia \\ favioshifres@gmail.com \\ Filiaciones institucionales \\ ${ }^{1}$ Laboratorio para el Estudio de la Experiencia \\ Musical, Facultad de Bellas Artes, Universidad \\ Nacional de La Plata (Argentina)
}

Mauro Hernán Valicente ${ }^{1}$

Nicolás Sicilia ${ }^{1}$

\section{Resumen}

Se presentan aportes al debate sobre la naturaleza abstracta o corporeizada de la mente. En particular se presenta el problema desde el punto de vista epistemológico, en torno a la determinación de la unidad de cómputo. Se muestra cómo la ciencia cognitiva clásica de la música exhibe un sesgo etnocéntrico en la selección de tal unidad al partir de la experiencia del sujeto eurocentrado. A tal fin se discuten aspectos de la relevancia de la unidad seleccionada en cuanto a su escala y perspectiva. Mediante ejemplos del campo de la psicología de la perfomance musical se argumenta que los enfoques corporeizados tienen más chances de evitar esos sesgos pues resuelven mejor el problema de la situacionalidad de los procesos cognitivos. Se concluye que la atención a la situacionalidad de los procesos cognitivos favorece la pluralidad epistémica en psicología de la música y por ende constituye un resguardo de justicia cognitiva.

\section{Palabras clave}

mente computacional | embodiment | epistemología | relevancia epistemológica | situacionalidad

Shifres, F., Valicente, M. H. y Sicilia, N. (2018). Contribuciones desde la experiencia musical al debate sobre la naturaleza de la mente. Revista de Psicología, 17(1), 51-68. doi: $10.24215 / 2422572 \mathrm{Xe} 014$
DOI

10.24215/2422572Xe014
Recibido

8 ene. 2018

Aceptado

19 mar. 2018

Publicado

27 jul. 2018

Editor

Nicolás Alessandroni | Facultad de Psicología, Universidad Autónoma de Madrid (España)
ISSN

2422-572X

Licencia

(c) Copyright: Shifres, F., et al. Este trabajo se distribuye bajo una licencia de Cultura Libre CC-BY 4.0

\section{Entidad editora}

RevPsi es una publicación de la

Facultad de Psicología (Universidad

Nacional de La Plata, Argentina)

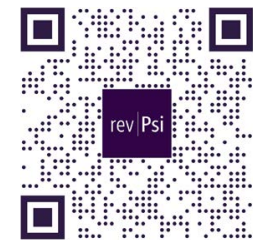

ACCESO ABIERTO DIAMANTE ○ 


\section{Contribuições da experiência musical para o debate sobre a natureza da mente}

\section{Resumo}

Contribuições para o debate sobre a natureza abstrata ou incorporada da mente são apresentadas. Em particular, o problema é apresentado do ponto de vista epistemológico, em torno da determinação da unidade de computação. Mostra como a ciência cognitiva clássica da música exibe um viés etnocêntrico na seleção de tal unidade a partir da experiência do sujeito eurocêntrico. Para este fim, aspectos da relevância da unidade selecionada em termos de sua escala e perspectiva são discutidos. Usando exemplos do campo da psicologia da performance musical, argumenta-se que as abordagens incorporadas são mais propensas a evitar esses vieses porque resolvem melhor o problema da situacionalidade dos processos cognitivos. Conclui-se que a atenção à situacionalidade dos processos cognitivos favorece a pluralidade epistêmica na psicologia da música e, portanto, constitui uma defesa da justiça cognitiva.

\section{Palavras-chave}

mente computacional | embodiment | epistemologia | relevância epistemológica | situacionalidade

\section{Contributions from the musical experience to the debate on the nature of the mind}

\section{Abstract}

Contributions to the debate on the abstract or embodied nature of the mind are presented. In particular, the problem is presented from the epistemological point of view, around the determination of the computing unit. It shows how classical cognitive science of music exhibits an ethnocentric bias in the selection of such a unit from the experience of the Eurocentric subject. To this end, aspects of the relevance of the unit selected in terms of its scale and perspective are discussed. Using examples from the field of the psychology of musical performance, it is argued that embodied approaches are more likely to avoid these biases because they better solve the problem of the situationality of cognitive processes. It is concluded that the attention to the situationality of the cognitive processes favors the epistemic plurality in the psychology of music and therefore constitutes a defense of cognitive justice.

\section{Keywords}

computational mind | embodiment | epistemology | epistemological relevance | situatedness 


\section{Aspectos destacados del trabajo}

- Se muestra la arbitrariedad de los enfoques cognitivos de la música basados en la teoría musical occidental.

- Se destacan las implicancias geo-corpo-socio-políticas del sesgo de los enfoques clásicos de la mente musical.

- Se problematiza la escala y la perspectiva epistemológica de los enfoques clásicos de la mente musical.

- Se destaca el abordaje de la situacionalidad de los procesos cognitivos por los enfoques corporeizados.

A lo largo del tiempo la curiosidad por conocer el modo en el que las personas comprendemos el mundo y actuamos en él de manera coherente respecto tanto de tal comprensión como de nuestros propios fines ha ocupado considerable atención de filósofos en primer lugar y luego de psicólogos, neurocientistas y biólogos, entre otros. Todos ellos han contribuido a delimitar y definir las principales categorías que permiten pensar estas cuestiones. Las ideas de mente, procesos cognitivos, percepción, conceptualización, juicio, y muchas más, fueron consolidando una base epistemológica sobre la cual abordar la problemática en un marco científico, esto es, de acuerdo con ciertos principios generales que validan el conocimiento construido. El modo en el que la ciencia moderna ha construido la noción de mente, ha permitido que las particularidades de dicha noción fuera ajustándose a modelos teóricos que, aunque referidos específicamente al entendimiento y la acción humanas, reflejan aspectos culturales mucho más generales de cada época. De tal manera, la noción de mente computacional es claramente un exponente del clima de época propio de la posguerra y la segunda mitad del siglo XX, afín al desarrollo tecnológico de dicho período y a las formas de conocimiento y control de los procesos sociales y culturales.

En ese contexto creció exponencialmente la investigación en el campo de la Psicología de la Música, un campo disciplinar que había nacido en el siglo XIX con los primeros laboratorios de psicología experimental, pero que había perdido impulso a la sombra de los movimientos psicológicos de finales del siglo XIX y la primera mitad del XX. Probablemente, el giro lingüístico operado en las ciencias humanas a partir de mediados del siglo pasado promovió sacudidas de similar intensidad (aunque de diferentes características) tanto en la psicología como en la musicología, facilitando su acercamiento, y transmitiendo esa intensidad a su cruce sobre el cual resurgió la disciplina.

En este trabajo, analizamos ese cruce en el momento de un nuevo giro en las ciencias humanas: el giro corporal, que desde hace un par de décadas viene empujando transformaciones importantes en las ciencias cognitivas, entre otras disciplinas. Nos 
proponemos reflexionar acerca del rol que el estudio de la experiencia musical puede tener en el debate acerca de la cognición entre los enfoques computacionales clásicos, y la segunda generación de ciencias cognitivas que adhiere a dicho giro corporal. Como en todo debate importante, las argumentaciones nunca pueden ser totalizadoras. Deliberadamente eludimos aquí los puntos más recorridos del debate: la discusión en torno al solipsismo y la cuestión representacional de la mente. Simplemente, presentamos una parte de la discusión que se vincula solamente a un aspecto de las teorías clásicas, la delimitación de la unidad de análisis, desde la convicción de poder contribuir al debate más amplio. Lo hacemos así, porque estimamos que a propósito de eso, la psicología de la música tiene reflexiones oportunas para hacer.

Pero además, lo hacemos así, porque nuestra intención es también política. Como también estamos convencidos de que todo debate epistemológico es un debate ideológico, que nos interpela acerca de nuestras creencias acerca del conocimiento y su origen y consecuencias sociales, situamos nuestro aporte en el marco de las críticas a la hegemonía del conocimiento social orientado por el paradigma de la ciencia positiva, y la subaltenización de otros modos de conocer al ser humano y su manera de construir el conocimiento acerca del mundo que lo rodea. En ese sentido, nuestra propuesta puede entenderse como parte del proyecto que procura poner en valor el conocimiento que adquiere relevancia en nuestro propio campo de actuación y es el resultado de las experiencias culturales y políticas del contexto que nos cobija. Esa relevancia puede resultar ininteligible en otros contextos porque las experiencias que busca rescatar y explicar no ocurren o son invisibilizados en ellos. Esta discusión, por lo tanto, no es abstracta para nosotros. Adquiere una dimensión concreta en el modo en el que nosotros transitamos nuestra propia experiencia y reconocemos la diversidad en otras experiencias.

En ese sentido, creemos que la psicología de la música puede contribuir a justipreciar la situacionalidad de cualquier modelo psicológico, porque la experiencia musical es ubicua a la vez que diversa, y son muy pocos los candidatos a componentes universales de dicha experiencia que resisten una crítica profunda como tales. Es por ello que la experiencia musical inspira ideas divergentes pudiendo contribuir a un debate epistemológico más amplio en el seno de las ciencias cognitivas de la música simplemente ofreciéndose como objeto de reflexión.

\section{La perspectiva de la mente computacional en la explicación de la experiencia musical}

La visión clásica de la cognición sostiene que le damos sentido a nuestra experiencia partiendo de la conceptualización del mundo basada en símbolos. Esto implica que tomamos del mundo que nos rodea información que es representada por tales símbolos. Esta es la base de los procesos cognitivos: los símbolos representan las unidades básicas de conocimiento, que por esa razón denominaremos aquí átomo de conocimiento. La teoría clásica sostiene que esos átomos pueden ser manipulados a través de algoritmos (procesos) por los cuales el sistema (la mente) los convierte en 
unidades de conocimiento de orden superior. Esta propuesta primordial tiene, entre otras, dos consecuencias muy importantes con relación a lo que nos proponemos tratar aquí. En primer lugar asume que el objeto de conocimiento puede entenderse como una estructura jerárquica de modo que la mente puede construirlo reconstruyendo esa estructura a partir de los elementos constituyentes básicos. En segundo lugar, implica que indagar en los procesos de construcción de conocimiento requiere que podamos definir, como punto de partida, la naturaleza y el alcance del átomo de conocimiento.

Así, el mundo es representado por símbolos sobre los que se opera para generar nuevos símbolos que se descodifican en nuestras respuestas en y para con el mundo. Todo lo que tiene luego entre la codificación y la descodificación de mundo es la mente, y como tal ocurre dentro de una caja negra de cuyo interior solamente podemos establecer hipótesis o modelos de funcionamiento. Más importante que entender la sustancia del cerebro es comprender la función de lo que tiene lugar allí (funcionalismo). Así se delimita el alcance de la teoría psicológica y se fijan las agendas de investigación.

El punto de partida de esas hipótesis o modelos es justamente la propuesta del átomo de conocimiento. En otros términos, todo modelo de procesamiento es construido a partir del establecimiento de la unidad simbólica. Los psicólogos que se ubican desde esta perspectiva la han sostenido entre otras cosas sobre el hecho de que los modelos de procesamiento, así como sus elementos constituyentes, se ajustan a las ideas que somos capaces de construir respecto de esos procesos y que intuitivamente podemos llevar al plano de la conciencia. "[L]as partes concientes e inconcientes de la mente son de la misma naturaleza esencial, construida con información y con los procesos que operan sobre ella" (Jackendoff, 1998, p. 35). Tomado como principio, esta afirmación legitima toda la lógica del procesamiento sobre la base de la consideración de una unidad de conocimiento que es la que emerge de la conciencia del investigador. En otras palabras, las unidades computacionales son establecidas a priori, por la conciencia del investigador, por el modo en el que el investigador concibe el objeto de conocimiento.

La estrategia de la ciencia cognitiva clásica se basa, como vemos, en dos premisas intuitivas: (i) la noción de que los procesos complejos son el resultado de la composición en términos reglados (gramáticas) de procesos más simples (de menor número de componentes); (ii) que el punto de partida de estos procesos simples es una unidad compatible con la intuición del investigador que formula el modelo, por lo que en general la asunción de dicho punto de partida no se cuestiona ni se investiga.

En este marco se considera que el modelo, en efecto, da cuenta de la realidad que pretende describir, cuando, entre otros requisitos es lógicamente plausible (Cohen, 2000). Esta plausibilidad depende tanto de la organización de los argumentos que sostienen el modelo como de la selección de sus componentes. Surgen, entonces, algunas preguntas: ¿cuáles son "las unidades intuitivas de la conciencia”? Y ¿de qué 
conciencia estamos hablando?

El estudio de la cognición musical comenzó identificando ese punto de partida en el sonido y sus atributos derivados de sus variables físicas: altura, intensidad, timbre, entre otros. Sin embargo, el giro lingüístico operado en la psicología a partir de la segunda mitad del siglo XX, requería de unidades de procesamiento compatibles con la noción de lenguaje (musical). De tal modo, los candidatos naturales a ser asumidos como puntos de partida incuestionados pasaron a ser los componentes prototeóricos de la Teoría Musical Occidental Moderna. Extendernos sobre esta idea nos alejaría innecesariamente del punto central de este trabajo, sin embargo, basta aquí con mencionar que se trata de constructos teóricos que proviniendo de las diferentes tradiciones teóricas que confluyeron en la Teoría Musical Occidental, tienen en común participar de manera decisiva en la consolidación y pedagogía de la notación musical occidental, con sus implicaciones en el campo performativo, compositivo y crítico/analítico. Algunos de ellos, que resultan útiles para definir lo que queremos analizar aquí son: la nota musical, el intervalo musical (entendido como distancia entre dos sonidos en un espacio virtual), la escala musical, las nociones escindidas de altura y ritmo como componentes básicos de la música, entre otros.

\begin{abstract}
Al escuchar música, no oímos los elementos como unidades desconectadas, sino unos en relación con otros. Los tonos individuales se perciben en términos de sus funciones en el contexto más amplio de la altura y el ritmo, y logramos un sentido de organización subyacente de la composición. La meta de la investigación en percepción musical, un área de estudio interdisciplinario de actividad creciente, es describir este proceso (Krumhansl, 1985, p. 371).
\end{abstract}

En este párrafo se identifica una serie de asunciones teóricas basadas en esas nociones teóricas fundamentales: la unidad de procesamiento es el tono individual (nota musical), que posee dos características primordiales, altura y duración, y que se vincula con otros para permitir la experiencia de coherencia. Además de dos preconceptos basados en la experiencia occidental moderna de la música: (i) la música se escucha, (ii) la música es compuesta. Se puede apreciar con claridad cómo tales conceptos resultan axiomáticos en la perspectiva computacional.

Decíamos que el modelo lingüístico propone que la realidad que la mente procesa se puede analizar como un lenguaje (computacional), lo que implica reconocer unidades atómicas que se organizar de manera jerárquica conforme reglas que integran grámáticas. Esto incluye a las cosas que nosotros hacemos a partir de comprender esa realidad. Así, el modelo identifica en nuestras respuestas "unidades atómicas que se organizan de manera jerárquica conforme reglas que integran gramáticas". Finalmente, el modelo asume que es posible establecer cierta correspondencia pragmática entre ambas organizaciones externas. Esas correspondencias son las que articulan los procesos que tienen lugar en el interior de la caja negra. La unificación de todo el modelo se garantiza por la lógica que vincula a las partes con el modelo teórico de base. De este modo, 
[e]n varios campos de la psicología cognitiva lidiamos con dos facetas del mundo: un sistema de reglas por un lado, y su reflejo en la mente pública. Algunos ejemplos incluyen, la base lógica de la teoría económica versus la toma de decisiones humana; la sintaxis versus la estructura del lenguaje hablado; las reglas de la geometría proyectiva versus la percepción de la perspectiva en las imágenes. En la musicología cognitiva (...) las dos facetas son las reglas de armonía tradicional occidental versus la habilidad del oyente para comprender el flujo de las notas, los acordes y las tonalidades (Kubovy, 1992, p. 199).

No está de más señalar que los sistemas que se mencionan en la cita, son sistemas que, aun partiendo de una instancia descriptiva, se instalan "en la mente pública" por su carácter regulativo. En particular, el sistema que nos incumbe, el de la armonía tradicional occidental, surge de las prácticas composicionales desarrolladas durante siglos en Europa que a su vez se vieron sujetas a principios canónicos que emergen de empresas teóricas (tratados) difundidos con el objeto de regularlas (véase Cook, 2002). De este modo, el pensamiento musical del oyente con experiencia en la música que proviene de dicha práctica ha sido modelado por el propio sistema regulativo. Se pone en evidencia que la proyección de una teoría musical, conforme la tradición teórica occidental (véase Christensen, 2002) como una "investigación sobre un campo de la capacidad cognitiva humana” (Lerdahl y Jackendoff, 1983, p. xi) es una tautología: el oyente aprende una serie de reglas y luego la investigación verifica que las aprendió, concluyendo en que ese sistema de reglas es "la capacidad cognitiva humana" y no la capacidad de aprenderlo. Esos sistemas de reglas establecen categorías por las cuales se considera y estudia el fenómeno. Esas categorías son propias del marco teórico, no del fenómeno, lo que implica que dan cuenta de la mirada particular que recibe el fenómeno. Al ser de ese modo malinterpretadas, convierten a esa mirada en la única mirada posible sobre el fenómeno y se convierten en categorías apriorísticas para el abordaje de cualquier fenómeno similar. Así, "el flujo de las notas, los acordes y las tonalidades" es confundido con el flujo de la música misma, y "las notas, los acordes y las tonalidades" emergen como categorías apriorísticas para abordar el estudio de la experiencia de ese flujo, en cualquier circunstancia. Esto es así, porque la lógica de los sistemas de reglas, imponen la necesidad, como decíamos antes, de partir de la unidad de información, el átomo de conocimiento, para elaborar un modelo de experiencia musical.

Nos proponemos aquí, entonces, examinar particularmente un aspecto de la crítica al modelo computacional de la mente que se refiere precisamente a la selección de la unidad de información, y a partir de dicha crítica señalar las ventajas que la perspectiva corporeizada de la cognición puede ofrecer para superarla. Con esto dejamos de lado enfoque críticos del modelo computacional que no resuelven las dicotomías clásicas (mente-cuerpo; percepción-acción; estímulo-respuesta, entre otras) de la que muchos enfoques corporeizados no se han desprendido. Pero además circunscribimos el análisis al dominio de la experiencia musical, que es el tema que convoca este número, debido a que nos permiten despegarnos de la lógica del pensamiento como lenguaje. 


\section{Claves epistemológicas en la delimitación de la unidad de conocimiento}

Originalmente las computadoras han funcionado procesando información que proviene de considerar la unidad representacional mínima posible conforme los recursos de traducción que el sistema posee. Pero, en la mente eso no necesariamente ocurre así. En este caso, la mínima unidad representación posible puede ser más bien el resultado de procesos cognitivos de variable complejidad, más que el origen de ellos. Por ejemplo, el pixel es una unidad informática de representación de información gráfica (visual). Obviamente, la mente no parte de ella. Los procesos visuales parten de considerar unidades relevantes, en términos de la experiencia visual del observador. Un ejemplo claro de esta diferenciación en música es la unidad de procesamiento métrico. La estructura métrica es una organización jerárquica de señales o impulsos psicológicas desenvueltos de manera regular en el transcurso del tiempo, que permite al oyente organizar la temporalidad del evento sonoro y medir su transcurrir según las unidades isócronas que dicha estructura establece. La estructura métrica tiene además el potencial de jerarquizar los eventos que resultan sincrónicos con aquellas señales psicológicas (pulsos). Los modelos computacionales de inferencia de la estructura métrica parten de tomar el mínimo intervalo de tiempo entre 3 o más ataques sucesivos isócronos (Temperley, 2001). Cuando escuchamos música tomamos para establecer el pulso de base 3 o más ataques isócronos sucesivos que no necesariamente son del mínimo intervalo de tiempo presente en el estímulo. Por el contrario, tomamos aquellos que resultan relevantes para nuestra escucha en ese momento determinado.

Esto nos lleva a cuestionar qué constituye la unidad de conocimiento que es simbolizada en el modelo computacional. ¿Cuán relevante es aquello que se hipotetiza como representación mental elemental? Vamos a sugerir que en la psicología clásica de la música lo que se hipotetiza como representación mental es lo que ya está representado, porque se toman los elementos que a lo largo de la evolución de la música europea, sus problemas de performance y su teoría, dieron lugar a la Notación Musical Occidental. En otros términos, la psicología clásica de la música toma como unidad de representación mental los elementos prototeóricos de la teoría musical occidental, sin orientar ningún esfuerzo a verificarla. Es axiomática. Como la notación musical considera como unidad sonora para ser anotada un segmento sonoro que resulta de una compleja relación entre el ataque y la extinción del sonido, con atención a la acción de ejecución (pulsar una cuerda, por ejemplo), y la definición categorial de su altura, y como sus cualidades a ser registradas justamente las de la altura y la duración, es la nota (escrita) la unidad de procesamiento que se toma de manera axiomática. La cita de Carol L. Krumhansl (1985) de arriba es más que elocuente en ese sentido. Así, el criterio de relevancia no problematizado, es el resultado de la co-evolución del pensamiento musical occidental con el sistema de notación musical, consolidada hacia el siglo XVII en la forma moderna de notación y el perfeccionamiento de un sistema musical tonal (que involucra la organización 
temporal mensurada según categorías proporcionales), propias del racionalismo dominante entonces. En otros términos, propia del sujeto occidental moderno, alfabetizado musical: el investigador.

Existe considerable evidencia musicológica e incipiente evidencia psicológica de que el tono y esos atributos, no constituyen la unidad experiencial de la música. Por ejemplo, María Inés Burcet (2014, 2015, 2017; Burcet y Uzal, 2017) ha problematizado la realidad cognitiva de la nota como unidad espontánea del pensamiento musical, indagando su ontogénesis. Sus trabajos muestran que en personas no alfabetizadas musicalmente, otras unidades, que no necesariamente tienen correlato notacional directo, tales como la pulsación métrica, el gesto musical o la extinción del sonido, poder ser tomadas como unidad de procesamiento en ciertas actividades musical. Asimismo muestra cómo otros atributos de tales unidades - el énfasis dinámico, la direccionalidad, la fuerza, etc. - resultan más importantes que la altura y la duración. Más aun, otros trabajos (Herrera 2014; Herrera y Shifres, 2011) muestran que incluso esa unidad excede el campo de lo sonoro y se presenta como una Gestalt multimodal habida cuenta de la relevancia que los oyentes dan al movimiento en la experiencia musical. En conjunto, tales estudios, entienden que las unidades de procesamiento que utiliza la perspectiva computacional son más bien aplicables al pensamiento musical alfabetizado e incluso gatilladas por la propia notación (Burcet y Shifres, 2011) y por lo tanto podría no dar cuenta de los procesos cognitivos de personas con otro background. De este modo, cada experiencia musical determina motivaciones de relevancia muy diversas, porque, básicamente ella está siempre orientada a una meta. El examen científico clásico de la cognición a menudo se desvincula de tales motivaciones e iguala al sujeto psicológico al sujeto de la situación experimental. Esta equivalencia implícitamente aceptada es prácticamente una condición de posibilidad del estudio científico, ya que:

Si sometiésemos la elección de objetivos a la discusión científica abierta y potencialmente infinita que caracteriza el análisis de objetos científicos, jamás seríamos capaces de establecer un criterio de relevancia coherente y llevar a buen término cualquier trabajo científico inteligible (De Sousa Santos, 2009, p. 66).

Este condicionamiento introduce una distorsión a sabiendas en la observación, que se acepta con el fin de acceder a problemas que son demasiado complejos para los instrumentos de observaciones de los que disponemos. Sin embargo, es muy importante considerar, siguiendo a Boaventura de Sousa Santos, que la relevancia así asumida es un disfraz epistemológico, que a menudo no lo vemos porque se nos torna invisible. "La invisibilidad del disfraz se basa en la credibilidad de la distorsión, y viceversa" (De Sousa Santos, 2009, p. 66). De este modo, en un contexto en el que la nota musical como unidad tenga gran credibilidad, como lo es el contexto académico en el que las personas aunque no sean músicos conocen la notación musical y hablan de la música en términos de su metalenguaje, no advertiremos suficientemente que este punto de partida es muy cuestionable epistemológicamente. Cuando este disfraz 
es menos creíble, porque nuestra experiencia choca contra él, entonces la distorsión aparece más clara. Por esa razón, los psicólogos de la música, trabajando en contextos que, a diferencia de los ambientes universitarios occidentales, se encuentran alejados de las lógicas de la teoría musical occidental, encontramos en las respuestas de nuestros participantes numerosos cuestionamientos a esos mismos principios de relevancia.

Los modelos idealizados (véanse Cassini, 2012 y Jones, 2005 en el campo científico en general, y Shifres, 2018 para una mirada en el campo de la música) a través de los cuales observamos nuestro objeto de estudio, favorecen la creación sistemática de ilusiones de correspondencia entre la experiencia y el propio modelo, por lo que las distorsiones se van haciendo así cada vez más invisibles (De Sousa Santos, 2009). Siguiendo esta lógica, la unidad se va tornando axiomática en la medida en que no reconocemos la escala que tomamos y la perspectiva que adoptamos.

Cuando observamos un hecho del mundo, lo podemos hacer a nivel de diferentes escalas. Los fenómenos que tienen lugar a cierta escala pueden ocurrir de otro modo a otra escala, o incluso pueden ser reemplazados por otros fenómenos. $\mathrm{Al}$ respecto, por poner un ejemplo, en la psicología de la música se ha discutido mucho si los experimentos que estudian la noción de consonancia a nivel de los intervalos (esto es, relaciones de dos sonidos), pueden dar cuenta de la experiencia de la consonancia en la audición de melodías. En esa línea, clásicamente, algunos investigadores argumentaron que escuchar una secuencia de tonos no puede ser visto simplemente como cuantitativamente diferente de escuchar una melodía en el contexto de la audición de un obra musical compleja. Concomitantemente, al describir los procesos de audición de la música tonal sobre una noción de tonalidad que examina la relación entre notas, acordes y escalas (Krumhansl, 1990) tomadas como unidades de procesamiento establecidas a priori (tonos), invisibiliza el problema de la escala y con ello oculta la distorsión del fenómeno que pretende observar. Volviendo al caso de la noción de consonancia un estudio de McDermott, Schultz, Undurraga y Godoy (2016) realizado en una comunidad Chiman de la Amazonia boliviana, muestra la distorsión de la valoración de la disonancia en contextos de tonos aislables, respecto de la irrelevancia que la consonancia tiene cuando se trata de la audición de las canciones propias de la comunidad.

Otro tipo de idealización/distorsión que los modelos presentan, (y es importante advertir) es el que proviene de la localización del sitio del observador, es decir de la perspectiva que adopta el observador. La perspectiva es un método de representación de la imagen en 3 dimensiones sobre un plano que se formaliza su base teórica en el Cinquecento y que permite comunicar la ilusión de la tercera dimensión. Consiste en la representación exacta de las dimensiones de lo observado desde un determinado punto conservando sus relaciones matemáticas a partir de ese punto. La ilusión se crea a expensas de la inmovilidad del punto vista (De Sousa Santos, 2009). Esto implica que si dirigimos la mirada desde otro punto, la representación se presenta claramente distorsionada. Cuando pensamos en la relevancia de una determinada 
categoría para asumirla como unidad de conocimiento, como en todo, adoptamos una determinada perspectiva. La validez de esa representación, estará supeditada entonces a la inmovilidad (unicidad) de dicho punto. La ciencia moderna asume que es posible posicionarse en un punto, a una distancia tal del fenómeno, que aunque nos movamos, la perspectiva no se vea sustancialmente alterada. Ese punto, que Santiago Castro Gómez (2005) denominó punto cero de observación, es el punto de observación de todo el universo. Cualquier pretensión de universalidad por lo tanto, invisibiliza la adopción de una determinada perspectiva. Siguiendo a De Sousa Santos (2009) podemos decir que cuando se oculta el punto de vista, la realidad de esta ilusión, es sustituida por una ilusión de la realidad y de ahí, la ilusión de la realidad pasa a ser la realidad de la realidad.

Como el punto cero es quimérico, la validez de la observación depende de cuán afín a nuestra experiencia sea el punto de observación real. En otros términos, y con relación a la unidad de conocimiento, la cuestión reside en ver en qué medida esa unidad es afín a nuestro sentido común. Por ejemplo, en un estudio sobre la evolución de los estilos musicales, Rodríguez Zivic, Shifres y Cecchi (2013) diseñaron un modelo que permitía calcular la probabilidad de que tuviera lugar un sonido $\mathrm{Z}$ dados dos sonidos consecutivos $(\mathrm{X}-\mathrm{Y}$ ), a lo largo de la melodía, tomando como $\mathrm{X}$ todos y cada uno de los sonidos de la melodía. El conjunto de X-Y-Z fue denominado trigrama. La idea estaba inspirada en el Modelo de Implicación-Realización (Krumhansl, 1995; Narmour, 1990), que propone que dado un intervalo melódico implicativo (X-Y), es más probable que ocurra un cierto intervalo realizativo (Y-Z) que cualquier otro. Sin embargo, el punto de vista adoptado por este estudio difería del de Narmour en que aquí un intervalo implicativo podía ser cualquier intervalo de la melodía (es decir podíamos partir de cualquier sonido), en cambio para la teoría, un intervalo implicativo tiene que responder a una serie de restricciones. Esas restricciones son afines al sentido común del oyente occidental, y el punto de observación está relativamente oculto en la teoría (véanse las críticas en Huron, 2006). En un ensayo crítico sobre los abordajes musicológicos a gran escala, Krumhansl (2015), señala esta diferencia de perspectiva marcando el alejamiento de la propuesta de Rodríguez Zivic et al. (2013) del sentido común de la noción de implicación-realización. En tal sentido, la crítica es por demás acertada: el trigrama es una unidad absolutamente contraintuitiva. Sin embargo, Rodríguez Zivic et al. (2013) analizaron un corpus de miles de obras musicales del repertorio europeo que abarcaban el período entre 1700 y 1930 y encontraron que la matriz general podía descomponerse en factores que se identificaban cada uno de ellos con las obras que correspondían a subperíodos dentro de ese lapso. Notablemente, esos sub-períodos se correspondían con los períodos históricos que la musicología denomina Barroco, Clasicismo, Romanticismo, Postromanticismo. El modelo permitió explicar diferencias entre los corpus de obras desde una perspectiva absolutamente contraituitiva con relación a la teoría occidental. La lógica de los trigramas es contraintuitiva, pero la presencia de unidades melódicas de varias notas (configuraciones temáticas, arpegios, diseños escalísticos, diseños interválicos lineales, etc.), brindaban a otra escala una explicación más convincente 
de los fenómenos que resultan arbitrarios en la escala nota a nota. Claramente, ese trabajo es una provocación epistemológica. Muestra como una unidad arbitraria, desvinculada de la experiencia musical de toda la tradición occidental, puede igual arrojar resultados que ajustan al modelo matemático. Es una muestra de que la coherencia entre las unidades y los resultados no dicen nada acerca de la realidad cognitiva de dichas unidades, solamente hablan de que son funcionales al modelo.

\section{La expresión en la performance musical y la oportunidad de las perspectivas corporeizadas de superar los problemas de la unidad}

En los estudios sobre ejecución musical expresiva y la comunicación entre ejecutantes y oyentes (Gabrielsson, 2003; Shaffer, 1976; Shifres, 2003), el átomo de conocimiento es la desviación expresiva. Ésta consiste en la modificación que el ejecutante realiza del valor de un determinado atributo del átomo universal (la nota). Específicamente la desviación expresiva es el valor de ese atributo correspondiente a una determinada nota tocada y el valor normalizado de esa nota. Por ejemplo, si el atributo estudiado es la dinámica expresiva (la intensidad del sonido o sonoridad), la unidad será calculada por la diferencia entre el valor de sonoridad real del sonido en cuestión y la sonoridad media de toda la ejecución. Así tendremos una medida de cuán fuerte o suave es tocada esa nota con relación a la media y por lo tanto se podrá medir el nivel de énfasis intencional que el ejecutante está aplicando sobre ella. El atributo de ejecución expresiva más estudiado es el timing que alude al alargamiento y acortamiento de los sonidos realizado intencionalmente con una finalidad expresiva (Shifres, 2014). La unidad de timing se toma por la diferencia entre la duración real de la nota tocada y el valor nominal de la duración de esa nota, generalmente tomado como la duración teórica que esa nota tendría que tener de acuerdo con la notación en la partitura. Así, la ciencia cognitiva clásica estudia el alargamiento o acortamiento de los sonidos en el curso de una interpretación musical como resultado (output) de un proceso por el cual el ejecutante comprende la estructura musical y proyecta su intención de comunicarla al oyente. En ese sentido, por ejemplo, un ritardando al final de una frase musical, indica un proceso por el cual el ejecutante comprende la estructura sintáctica de la pieza y la comunica al oyente, sobre la base que éste conoce la regla generativa que adjudica buena forma a un final de frase ritardado. De acuerdo con esta idea, la decisión del ritardando final, obedeciendo a tal regla, es independiente de las circunstancias que rodean la ejecución. Así, una ejecución expresiva puede ser simulada por una computadora que haya sido adecuadamente programada conforme tales reglas generativas (Sundberg, 1993). Desde esta perspectiva, la unidad de medición de la expresión en la performance es tomada a priori, independientemente de las circunstancias del hecho comunicacional (la performance en sí), del cuerpo que ejecuta esa frase, su fisiología, su tamaño, su ubicación, su biografía, además de su contexto cultural, su historia, etc.

Sin embargo, en dos últimas décadas numerosos estudios han destacadola importancia del cuerpo en las particularidades de la ejecución (Davidson, 2001; Wanderley, 
Vines, Middleton, McKay y Hatch, 2005), y su procesamiento independiente de las representaciones mentales (Schiavio y Høffding, 2015; Shifres, 2009; Shifres, Pereira Ghiena, Herrera y Bordoni, 2012; Walton et al., 2018). La perspectiva cognitiva clásica dice que existe una relación generativa entre la estructura musical, tal como la entiende el ejecutante y sus acciones en la ejecución (por ejemplo alargamiento o acortamiento de una nota). De este modo, la desviación expresiva es la unidad de conocimiento a partir de la cual se construye el modelo del proceso (por ej. Palmer, 1989; Sloboda, 1983).

Siguiendo esa idea, medimos los alargamientos y acortamientos de las notas en una ejecución de tango Malena de Homero Manzi y Lucio Demare interpretada por Roberto Goyeneche en 1987 (Shifres, 2015). En los versos "a yuyo del suburbio / su voz perfuma", Goyeneche alarga y acorta ciertas notas que rompen con el fraseo esperado, alargando notablemente la nota en la que canta la palabra voz. Encontramos además que la cantante Adriana Varela, que explícitamente reconoce la influencia de Goyeneche sobre su propio estilo de ejecución, canta en una actuación de 2012 con un fraseo muy similar. Nos preguntamos, entonces, de qué modo Varela construyó su ejecución. La perspectiva cognitiva clásica nos conducía a tomar la desviación temporal de la nota como unidad, y por lo tanto a modelizar un perfil de desviaciones temporales de la performance de Goyeneche de la frase completa que Varela estuviera replicando con notable fidelidad. Esto implicaría que ella es capaz de construir una representación interna de dicho perfil que se activa en el momento de la ejecución. Recordemos que la unidad es el alargamiento de la nota, y que fue fijada a priori, antes de observar la ejecuciones de Goyeneche y de Varela.

Sin embargo al examinar otras actuaciones de Varela del mismo tango encontramos que, si bien muchas veces replicaba el perfil de Goyeneche, muchas otras no. En estas últimas, el fraseo se organizaba en acuerdo con la sintaxis de la frase musical. Nos preguntamos, qué podría estar modelizando esa alternativa. ¿Por qué en algunas situaciones ocurría una cosa y en otras, otra? Pero además, si Varela explícitamente reconoce la influencia de Goyeneche en su interpretación, pero sus versiones distan mucho de ser una simple réplica o imitación del perfil de timing de él, y varían de situación en situación ¿a través de qué unidades de conocimiento circula esta noción de estilo entre ellos? En otros términos ¿Cómo construye Varela el estilo que no es una imitación de Goyeneche pero que se pronuncia como heredero de él? ¿Puede la desviación expresiva tomada a priori como unidad de información explicar eso? Evidentemente, hay una adecuación al momento, que escapa a la idea del modelado computacional.

En ese sentido, el denominado giro corporal en psicología puede brindar algunas pistas que permitan entender mejor cada situación. Según esta perspectiva, el conocimiento no se construye a través del procesamiento de información simbólica, es decir como representaciones de hechos y eventos del entorno en formato proposicional, sino a través del modo en el que interactuamos con y en él (enacción). Así,

La cognición es lo que tiene lugar cuando el cuerpo se engarza en el mundo cultural y físico y debe ser estudiado en términos de las interacciones dinámicas 
entre las personas y el entorno ... No debemos suponer que la cognición sea puramente interna, simbólica, computacional e incorpórea; por el contrario, debemos buscar maneras tanto generales como detalladas en las que el lenguaje y el pensamiento están inextricablemente conformados por la acción corporeizada (Gibbs, 2006, p. 9).

A partir de esta idea, ampliamos el foco de la observación. No solamente atendimos a las desviaciones del timing de las performances, sino que observamos el contexto de la actuación (escenario, músicos que acompañaban, intervenciones del público, etc.) intentando encontrar en el contexto algún dato. Buscábamos encontrar algún dato en lo que Rohlfing, Rehm y Goecke (2003) llaman situacionalidad. De acuerdo con estos autores los procesos cognitivos dependen de la vinculación del sistema cognitivo con el mundo, y éste, a su vez, se ve modificado por ellos. De ahí que un organismo no responde a los estímulos siguiendo un programa de acción (como lo asumía el enfoque cognitivista clásico), sino que su modo de responder dependerá de cómo interactúa con la situación y con el contexto. La situación es el entorno físico del aquí $\mathrm{y}$ ahora del proceso, y el contexto, es un complejo que involucra tanto un nivel global, de carácter socio-cultural, por ejemplo la cultura musical de pertenencia, como un nivel local, por ejemplo una actuación en concierto. Situación y contexto configuran la situacionalidad.

Paralelamente examinamos diferentes versiones del propio Goyeneche del mismo tango y observamos que la particularidad de ese fraseo no fue siempre así. Por el contrario, puede observarse una elaboración de ese fraseo a lo largo de por lo menos 20 años. Notablemente encontramos que Goyeneche elabora un gesto con su mano izquierda, que puede entenderse como gesto metafórico (McNeill, 1992) que alude al aroma y la acción de perfumar, dentro del tipo de gestualidad relativamente contenida que realiza con sus manos. Ese gesto va co-evolucionando con la duración de la sílaba $\mid v o z \backslash$ (que antecede a \perfuma \en el verso). En otros términos, la duración de \voz $\backslash$ no parece estar motivada por ninguna razón estructural musical, sino más bien por la necesidad de dar lugar a expresar gestualmente lo que sigue, generando cierta expectativa.

Sugestivamente, notamos que el timing de Varela se asemeja al de Goyeneche cuando espontáneamente realiza con su mano izquierda un gesto similar al de él. Cuando su expresión gestual se diferencia de la de Goyeneche, el timing es también diferente. Esta diferencia obedece a la situacionalidad de la performance, por un lado el modo en el que el entorno físico le permite realizar espontáneamente ese gesto (por ejemplo, en una actuación se apoya en el pie del micrófono con la mano izquierda, por lo tanto, ésta no le queda libre para realizar el gesto), por otro al contexto global y local de la performance (por ejemplo algunas interpretación presentan un carácter deciso y los gestos que viene realizado con la mano izquierda se vinculan con esa fuerza de decisión -al decir "en cada verso pone", destaca con un gesto contundente la palabra \pone -, y preparan la posición del cuerpo y el brazo para el gesto de (perfuma\). 
Pero lo más relevante para lo que estamos exponiendo aquí es que la unidad de conocimiento por la cual podríamos modelizar el estilo de Goyeneche y su relación con el de Varela no era la nota sino una Gestalt sonoro-kinético-conceptual que es posible solamente bajo ciertas condiciones del contexto. Es decir que fue la observación de esos fenómenos en su particularidad, sus cuerpos, sus contextos, lo que permitió determinar un posible núcleo de conocimiento construido/transmitido. De ahí, que se parte de una restricción corporo-contextual en el modelado del proceso.

En este trabajo ensayamos algunas ideas acerca de cómo el estudio de la experiencia musical puede contribuir a una crítica de la perspectiva cognitivista clásica de mente. Si bien esta perspectiva clásica viene siendo criticada en muchos aspectos, principalmente el problema de la representación y el internalismo. Nuestra crítica apunta a la pretensión de universalidad de sus hallazgos a partir de invisibilizar el sesgo geo-corpo-socio-político de los axiomas en los que se basa. De tal modo, el rescate que hacemos de las ciencias cognitivas de segunda generación, que proponen una cognición corporeizada y situada, no se enmarca directamente en la discusión acerca de aquellos tópicos. Sin embargo, se vincula de manera elíptica con ellos porque la atención al cuerpo y al entorno, y la incontrovertible existencia de una incalculable diversidad de cuerpos y entornos, nos permite poner el foco sobre las consecuencias sociales de la mirada universalista de la psicología. El estudio del compromiso corporal pone el foco de la atención en el propio sujeto y no en el resultado de la tarea asignada a partir de hipótesis que modelizan intuiciones.

Como señala De Sousa Santos (2003), la ciencia contemporánea nos invita a cambiar la perspectiva que heredamos de la física clásica, porque

[e]n vez de eternidad, tenemos la historia; en vez de determinismo, la imprevisibilidad; en vez del mecanicismo, la interpenetración, la espontaneidad y la auto-organización; en vez de reversibilidad, la irreversibilidad y la evolución; en vez del orden, el desorden; en vez de la necesidad, la creatividad y el accidente (De Sousa Santos, 2003, p. 77).

Atender al cuerpo y a la situación evita el sesgo que proviene de enunciar categorías a priori que se vinculan más con las experiencias de los cuerpos y situaciones del sujeto occidental moderno y que, como tratamos de mostrar, resultan por ello arbitrarias en su pretensión de universales. El átomo de conocimiento universal es quimérico porque el conocimiento es complejo, y los procesos involucrados en él deben serlo también. La ciencia contemporánea cuestiona, cada vez más, la noción de leyes simples gobernando tanto los procesos naturales como los sociales. Esa simplificación, en su afán de permitir la inteligibilidad del fenómeno que estamos abordando puede ocultarnos dimensiones del problema que pueden ser interesantes. Así, incluso en el marco de un planteo internalista y representacional, tal atención hace posible que las hipótesis de la caja negra resultan menos distorsionadas porque atienden al sujeto, su situación y su historia. Cada persona se encuentra en la experiencia del mundo de un modo particular, y en su entendimiento tiene lugar su propia historia. 
La idea de que la mente humana procede según las intuiciones del sujeto moderno occidental y la naturalidad con la que se aceptan esas distorsiones son en principio sospechosamente etnocéntricas. Los procesos cognitivos, aun de base simbólica, no tienen por qué ser todos iguales. Tratamos de mostrar el propio punto de partida de ellos puede ser diferente del que intuimos, porque ellos tienen una historia particular, que se vinculan con la del individuo, su biografía, su lugar, su manera de estar en el mundo. La psicología clásica de la música recoge la biografía, el lugar y el modo de estar en el mundo del sujeto moderno, cuya cultura construyó, a lo largo de los siglos, un modo particular de representar la música, y elaboró un hacer musical afín a dicho modo. Es el sujeto que al pensar en música piensa en notas, cuyas dimensiones más relevantes son la altura y el ritmo, que conforman melodías. Las teorías cognitivas musicales, son teorías de los procesos cognitivos del sujeto en laboratorio, examinado bajo los principios formales que rigen la notación musical. Estas teorías resultan impotentes para explicar la musicalidad emergente en el vínculo madre-hijo o las habilidades musicales de un niño en una cuerda de candombe (Shifres, 2014).

Más allá de los tópicos del internalismo y la representación, el cognitivismo clásico es blanco de la crítica a la ciencia moderna en general, y esto involucra fundamentalmente los criterios de validez del conocimiento que emergen de sus bases racionales. Las abstracciones de cálculos de cómputos, terminan siendo condioncionamientos epistemológicos, es decir restricciones del campo impuestas por una serie de prejuicios que operan en el propio sistema teórico del investigador. Por ejemplo, la necesidad que la perspectiva clásica tiene de cuantificar las dimensiones que se pueden identificar dentro de un determinado problema y someter dicha cuantificación a lógicas matemáticas ya están imponiendo una perspectiva que inhibe cualquier otra mirada del problema. Así, del mismo modo que con la selección de la unidad de conocimiento, estos criterios de validez no necesariamente son compatibles con nuestras experiencias y necesidades. Sin embargo, si la psicología, en vez de mirar hacia adentro de su lógica con la ambición, propia del pensamiento hegemónico, de comprobar su universalidad (como superioridad), se preocupara más por la diversidad tal vez podría dar cabida a respuestas diferentes a las preguntas que nos planteamos respecto de la experiencia humana, y no solamente aquellas que satisfacen su propia experiencia. En esa pluralidad la psicología podría estar trabajando por la justicia cognitiva.

\section{Referencias}

Burcet, M. I. (2014). Realidad perceptual de la nota como unidad operativa del pensamiento musical (tesis de maestría). Universidad Nacional de La Plata, La Plata.

Burcet, M. I. (2015). Las unidades de la escritura musical como categorías para pensar la música. En F. Shifres y P. Holguín (Eds.), El desarrollo de las habilidades auditivas de los músicos. Teoría e investigación (pp. 153-184). La Plata: GITeV.
Burcet, M. I. (2018). Hacia una epistemología decolonial de la notación musical. Revista Internacional de Educación Musical, 5, 129-138. HTTPS://DOI.ORG/10.12967/RIEM-2017-5

Burcet, M. I. y Shifres, F. (2011). Selección y uso de unidades musicales en una tarea de transmisión oral. En R. Herrera y M. I. Burcet (Eds.), Musicalidad humana: debates actuales en evolución, desarrollo y cognición e implicancias socio-culturales (pp. 703-710). Buenos Aires: SACCoM. 
Burcet, M. I. y Uzal, S. (2017). La representación de la altura musical: Un estudio con adultos en etapas iniciales de la adquisición de la notación musical. En N. Alessandroni y M. I. Burcet (Eds.), La experiencia musical. Investigación, interpretación $y$ prácticas educativas (pp. 105-115). Buenos Aires: SACCoM.

Cassini, A. (2012). Idealización, abstracción y proliferación de modelos. Epistemología e Historia de la Ciencia, 18, 130-136.

Castro-Gómez, S. (2005). La hybris del punto cero: Ciencia, raza e ilustración en la Nueva Granada (1750-1816). Bogotá: Pontificia Universidad Javeriana.

Christensen, T. (2002). Introduction. En T. Christensen (Ed.), The Cambridge history of Western music theory (pp. 1-23). Cambridge: Cambridge University Press.

Cohen, G. (2000). Hierarchical models in cognition: Do they have psychological reality? European Journal of Cognitive Psychology, 12(1), 1-36. HTTPS://DOI.ORG/10.1080/095414400382181

Cook, N. (2002). Epistemologies of music theory. En T. Christensen (Ed.), The Cambridge history of Western music theory (pp. 78-105). Cambridge: Cambridge University Press.

Davidson, J. W. (2001). The role of the body in the production and perception of solo vocal performance: A case study of Annie Lennox. Musicae Scientiae, 5(2), 235-256. HTTPS://DOI. ORG/10.1177/102986490100500206

De Sousa Santos, B. (2003). Crítica de la razón indolente: Contra el desperdicio de la experiencia (Vol. 1). Bilbao: Desclée de Brouwer.

De Sousa Santos, B. (2009). Una epistemología del sur: La reinvención del conocimiento y la emancipación social. México D.F.: Siglo XXI.

Gabrielsson, A. (2003). Music performance research at the millennium. Psychology of Music, 31(3), 221-272. HTTPS://DOI. ORG/10.1177/03057356030313002

Gibbs, R. W. (2006). Embodiment and cognitive science. Cambridge: Cambridge University Press.

Herrera, R. (2014). La representación de la altura musical en la notación: Procesos cognitivos implicados (tesis de maestría). Universidad Nacional de La Plata, La Plata.
Herrera, R. y Shifres, F. (2011). La construcción espontánea de la representación temporal. En A. Pereira Ghiena, P. Jacquier, M. Valles y M. Martínez (Eds.), Musicalidad humana: Debates actuales en evolución, desarrollo y cognición e implicancias socio-culturales (pp. 747-756). Buenos Aires: SACCoM.

Huron, D. B. (2006). Sweet anticipation: Music and the psychology of expectation. Cambridge: MIT press.

Jackendoff, R. S. (1998). La conciencia y la mente computacional (A. A. Gumiel, Trad.). Madrid: Visor.

Jones, M. R. (2005). Idealization and abstraction: A framework. En M. R. Jones y N. Cartwright (Eds.), Idealization XII: Correcting the model. Idealization and abstraction in the sciences (pp. 173-217). Amsterdam/New York: Rodopi.

Krumhansl, C. L. (2015). Statistics, structure, and style in music. Music Perception: An Interdisciplinary Journal, 33(1), 20-31. нтTPs:// DOI.ORG/10.1525/MP.2015.33.1.20

Krumhansl, C. L. (1995). Music psychology and music theory: Problems and prospects. Music Theory Spectrum, 17(1), 53-80.

Krumhansl, C. L. (1985). Perceiving tonal structure in music: The complex mental activity by which listeners distinguish subtle relations among tones, chords, and keys in Western tonal music offers new territory for cognitive psychology. American Scientist, 73(4), 371-378.

Kubovy, M. (1992). Reviewed work: Cognitive foundations of musical pitch by Carol L. Krumhansl. American Scientist, 80(2), 199-199.

Lerdahl, F. y Jackendoff, R. S. (1983). A generative theory of tonal music. Cambridge: MIT press.

McDermott, J. H., Schultz, A. F., Undurraga, E. A. y Godoy, R. A. (2016). Indifference to dissonance in native Amazonians reveals cultural variation in music perception. Nature, 535(7613), 547-550. HTTPS://DOI.ORG/10.1038/NATURE18635

McNeill, D. (1992). Hand and mind: What gestures reveal about thought. Chicago/Londres: University of Chicago press.

Narmour, E. (1990). The analysis and cognition of basic melodic structures: The implicationrealization model. Chicago: University of Chicago Press. 
Palmer, C. (1989). Mapping musical thought to musical performance. Journal of Experimental Psychology: Human Perception and Performance, 15(12), 331-346.

Rodriguez Zivic, P. H., Shifres, F. y Cecchi, G. A. (2013). Perceptual basis of evolving Western musical styles. Proceedings of the National Academy of Sciences, 110(24), 10034-10038. HTTPS://DOI.ORG/10.1073/PNAS.1222336110

Rohlfing, K., Rehm, M. y Goecke, K. U. (2003). Situatedness: The interplay between context(s) and situation. Journal of Cognition and Culture, 3(2), 132-156. HTTPS://DOI. ORG/10.1163/156853703322148516

Schiavio, A. y Høffding, S. (2015). Playing together without communicating? A pre-reflective and enactive account of joint musical performance. Musicae Scientiae, 19(4), 366-388. HTTPS://DOI. ORG/10.1177/1029864915593333

Shaffer, L. H. (1976). Intention and performance. Psychological Review, 83(5), 375-393. HTTPs://DOI. ORG/10.1037/0033-295X.83.5.375

Shifres, F. (2003). La cooperación interpretativa de ejecutantes y oyentes. Hacia un modelo interpretativo de la ejecución musical. En G. Ferreira y L. Bruno (Eds.), Actas del IX Congreso de la Sociedad Argentina de Lingüística. Córdoba: Universidad Nacional de Córdoba.

Shifres, F. (2009). Movement and the practice of meaning in song. En C. Laurier, O. Lartillot, T. Eerola y P. Toiviainen (Eds.), Proceeding of the 7th Triennial Conference of European Society for the Cognitive Sciences of Music (pp. 495-502). Jyväskylä: ESCOM.

Shifres, F. (2014). Algo más sobre el enlace entre la infancia temprana y música: El poder expresivo del rubato. En S. A. Español (Ed.), Psicología de la música y del desarrollo (pp. 21-70). Buenos Aires: Paidós.
Shifres, F. (2015). Los desafíos epistemológicos de la cognición corporeizada a la pedagogía musical. En O. Grau, F. Ortega, G. Celedón y E. Oyarzún (Eds.), La instancia de la música (pp. 113-142). Santiago de Chile: Universidad Metropolitana de Ciencias de la Educación.

Shifres, F. (2018). Realidad e idealización del dominio de la notación musical. Revista Foro de Educación Musical, Artes y Pedagogía, 3(4), 13-44.

Shifres, F., Pereira Ghiena, A. , Herrera, R. y Bordoni, M. (2012). Estilo de ejecución musical y de danza en el tango: Atributos, competencia y experiencia dinámica. Cuadernos de Música, Artes Visuales y Artes Escénicas, 7(2), 83-108.

Sloboda, J. A. (1983). The communication of musical metre in piano performance. The Quarterly Journal of Experimental Psychology Section A, 35(2), 377-396. HTTPS://DOI. ORG/10.1080/14640748308402140

Sundberg, J. (1993). How can music be expressive? Speech Communication, 13(1-2), 239-253. HTTPS://DOI.ORG/10.1016/0167-6393(93)90075-V

Temperley, D. (2001). The cognition of basic musical structures. Cambridge/Londres: MIT Press.

Walton, A. E., Washburn, A., Langland-Hassan, P., Chemero, A., Kloos, H. y Richardson, M. J. (2018). Creating time: Social collaboration in music improvisation. Topics in Cognitive Science, 10(1), 95-119. HTTPS://DOI.ORG/10.1111/TOPS.12306

Wanderley, M. M., Vines, B. W., Middleton, N., McKay, C. y Hatch, W. (2005). The musical significance of clarinetists' ancillary gestures: An exploration of the field. Journal of New Music Research, 34(1), 97-113. HTTPs://DOI. ORG/10.1080/09298210500124208 